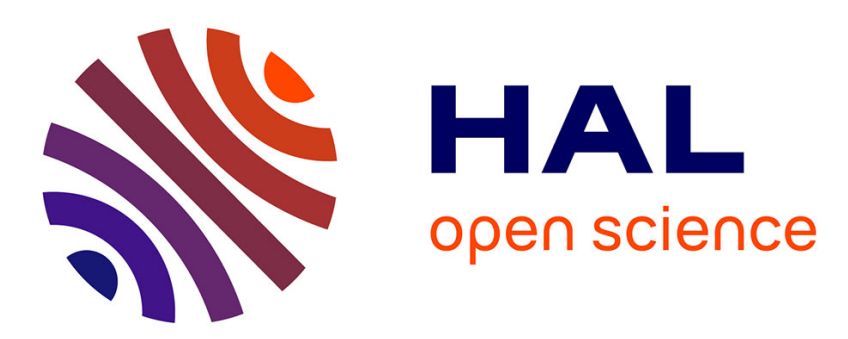

\title{
Atténuation des ondes ultrasonores par les dislocations dans la glace I h. Application à l'étude de la plasticité
}

\author{
J. Tatibouet, C. Mai, J. Perez, R. Vassoille
}

\section{To cite this version:}

J. Tatibouet, C. Mai, J. Perez, R. Vassoille. Atténuation des ondes ultrasonores par les dislocations dans la glace I h. Application à l'étude de la plasticité. Revue de Physique Appliquée, 1980, 15 (4), pp.835-842. 10.1051/rphysap:01980001504083500 . jpa-00244792

HAL Id: jpa-00244792

https://hal.science/jpa-00244792

Submitted on 1 Jan 1980

HAL is a multi-disciplinary open access archive for the deposit and dissemination of scientific research documents, whether they are published or not. The documents may come from teaching and research institutions in France or abroad, or from public or private research centers.
L'archive ouverte pluridisciplinaire HAL, est destinée au dépôt et à la diffusion de documents scientifiques de niveau recherche, publiés ou non, émanant des établissements d'enseignement et de recherche français ou étrangers, des laboratoires publics ou privés. 


\title{
Atténuation des ondes ultrasonores par les dislocations dans la glace $\mathbf{I}_{h}$
}

\section{Application à l'étude de la plasticité}

\author{
J. Tatibouet, C. Mai, J. Perez et R. Vassoille \\ I.N.S.A., Groupe d'Etudes de Métallurgie Physique et Physique des Matériaux \\ E.R.A. no 463, Bât. 502, 69621 Villeurbanne Cedex, France \\ (Reçu le 18 octobre 1979, révisé le 11 janvier 1980, accepté le 11 janvier 1980)
}

\begin{abstract}
Résumé. - Des mesures d'atténuation d'ondes ultrasonores ont été effectuées sur des monocristaux de glace avant et après déformation plastique, en fonction de la fréquence, de l'amplitude de l'onde ultrasonore, ou bien pendant l'application d'une contrainte quasi statique. Des mesures ont également été faites pendant la déformation plastique. Deux modèles de dissipation d'énergie par les dislocations ont été développés : ceux-ci reposent sur l'hypothèse du mouvement de décrochements géométriques ou bien du déplacement à courte distance du cœur des dislocations supposé non cristallin; ces deux modèles sont appliqués respectivement aux mesures à basse et haute température. Nos résultats et l'analyse proposée permettent la reconstitution théorique des courbes effort-déformation présentant bien les caractéristiques observées.
\end{abstract}

\begin{abstract}
The attenuation of ultrasonic waves in ice single crystals before and after plastic deformation have been measured in function of the frequency of the amplitude of ultrasonic waves and during the application of a quasi static stress. Measurements have been done also when specimens were plastically strained. Two models of energy dissipation by dislocations in ice have been developed assuming the movement of geometrical kinks or the displacement at short distance of the dislocation core supposed to be non crystalline, these models are applied to low and high temperature measurements respectively. The application of our results leads to the building of theoretical stress-strain curves in ice showing the observed features.
\end{abstract}

1. Introduction. - Nous avions déjà effectué des mesures de caractéristiques ultrasonores sur la glace $I_{h}$ [1]. En particulier nous avions mis en évidence une augmentation de l'atténuation après déformation plastique (Fig. 1).

Le comportement en fonction de la fréquence de l'onde ultrasonore avait également été étudié à $100 \mathrm{~K}$ (Fig. 2). De tels comportements avaient été attribués à la dissipation d'énergie ultrasonore par les dislocations et à la variation de densité de celles-ci lors de la déformation plastique. Nous avons donc complété nos résultats en faisant varier l'amplitude de l'onde ultrasonore, en imposant une contrainte quasi statique et en regardant de plus près l'effet de la température aux hautes fréquences pour essayer de préciser un modèle de dissipation d'énergie plus approprié que les modèles classiques (corde vibrante, décrochements géométriques) au cas de la glace. Il apparaît alors possible d'appliquer les mesures ultrasonores à l'étude de la plasticité de la glace.

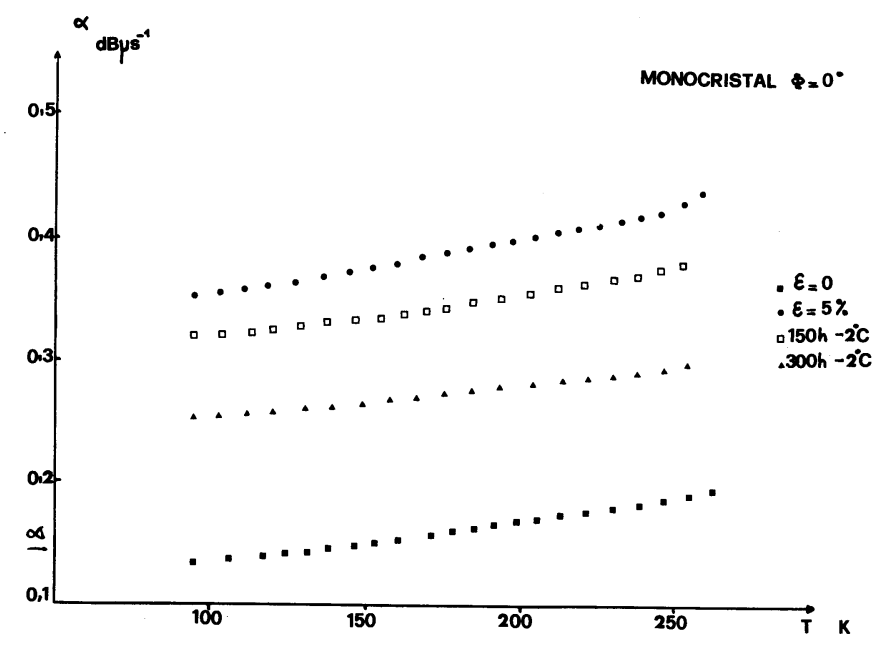

Fig. 1. - Variation de l'atténuation en fonction de la température, influence de la déformation plastique et des recuits à $271 \mathrm{~K}$. Fréquence d'essai $5 \mathrm{MHz}$.

[Attenuation versus temperature. Influence of plastic deformation and annealing at $271 \mathrm{~K}$. Test frequency $5 \mathrm{MHz}$.] 


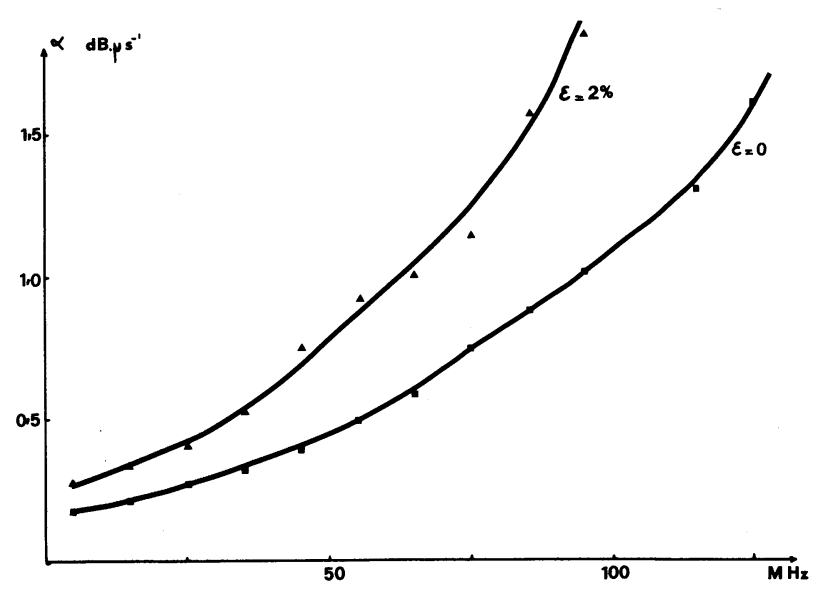

Fig. 2. - Variation de l'atténuation en fonction de la fréquence de l'onde ultrasonore à $100 \mathrm{~K}$.

[Attenuation versus frequency at $100 \mathrm{~K}$.]

2. Techniques expérimentales. - Les caractéristiques ultrasonores de la glace ont été étudiées par méthode d'écho pulsé. L'appareillage comprend un ensemble MATEC 9000, un appareillage permettant des mesures automatiques d'atténuation et de vitesse et un cryostat à température variable entre $80 \mathrm{~K}$ et $273 \mathrm{~K} \mathrm{[2].} \mathrm{Cet} \mathrm{ensemble} \mathrm{est} \mathrm{couplé} \mathrm{avec} \mathrm{une}$ machine de traction compression INSTRON permettant des mesures pendant la déformation même tant que celle-ci ne dépasse pas quelques pourcents.

L'installation permet des mesures d'atténuation

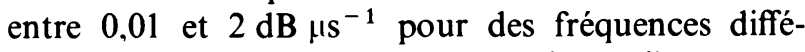
rentes obtenues à partir des harmoniques d'un quartz de polarisation longitudinale et de fréquence fondamentale $5 \mathrm{MHz}$.

Les monocristaux de glace ont été fournis par le laboratoire de Glaciologie de Grenoble. Ils sont taillés mécaniquement pour obtenir un parallélépipède de $15 \times 15 \times 40 \mathrm{~mm}^{3}$. Les faces sont, à l'aide d'un gabarit, rectifiées et polies sur soie afin d'obtenir un parallélisme et une planéité permettant des mesures correctes à hautes fréquences. Le liant entre échantillon et quartz est du méthyl 4-1 pentène.

Les échantillons sont déformés par fluage en compression à la température de $263 \mathrm{~K}$. Les faces intéressant la mesure sont à nouveau rectifiées et repolies après déformation.

3. Résultats. - 3.1 EFFET DE L'AMPLITUde DE L'ONDE ULTRASONORE. - Nous avons effectué des mesures d'atténuation en fonction de l'amplitude de l'onde ultrasonore. La mesure de l'amplitude de l'onde ultrasonore a été effectuée par l'intermédiaire de la mesure, à l'aide d'un oscilloscope haute impédance d'entrée, de la tension crête à crête de l'impulsion électrique excitant le quartz émetteur. Les mesures ont été faites à $5 \mathrm{MHz}$ sur des échantillons déformés et non déformés. La figure 3, sur laquelle est portée la variation $\Delta \alpha$ de l'atténuation entre l'échantillon non déformé et déformé en fonction

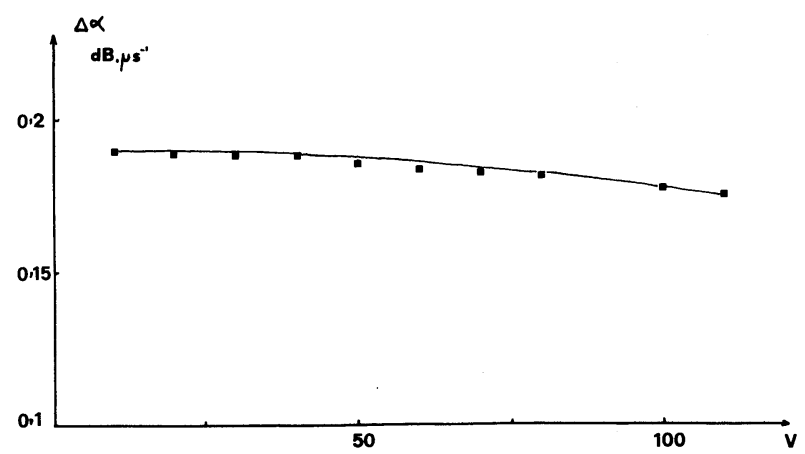

Fig. 3. - Variation de l'atténuation avec l'amplitude de l'onde ultrasonore $(T=258 \mathrm{~K}) \Delta \alpha=\alpha_{\text {déformé }}-\alpha_{\text {non déformé }}$.

[Attenuation variation versus ultrasonic wave amplitude $\left.(T=258 \mathrm{~K}) \Delta \alpha=\alpha_{\text {strained }}-\alpha_{\text {initial. }}\right]$

de la tension appliquée au quartz émetteur, montre une légère diminution de l'accroissement d'atténuation lorsque l'amplitude de l'onde ultrasonore augmente. Si le modèle de la corde vibrante exclut une telle variation, le modèle de Suzuki ét Elbaum prévoit, associé à l'empilement des décrochements, une diminution de l'atténuation, mais pour une amplitude plus élevée que celle mise en œuvre dans notre expérience.

3.2 EFfet De la température sur la Relation ENTRE $\Delta \alpha$ ET LA FRÉQUENCE. - Le résultat de la figure 4 correspond à la variation d'atténuation due à la déformation plastique en fonction de la fréquence et à 2 températures $(100 \mathrm{~K}$ et $260 \mathrm{~K})$. Nous ne remarquons un effet de la température que vers les hautes fréquences pour lesquelles la variation d'atténuation à $260 \mathrm{~K}$ est légèrement inférieure à celle obtenue à $100 \mathrm{~K}$.

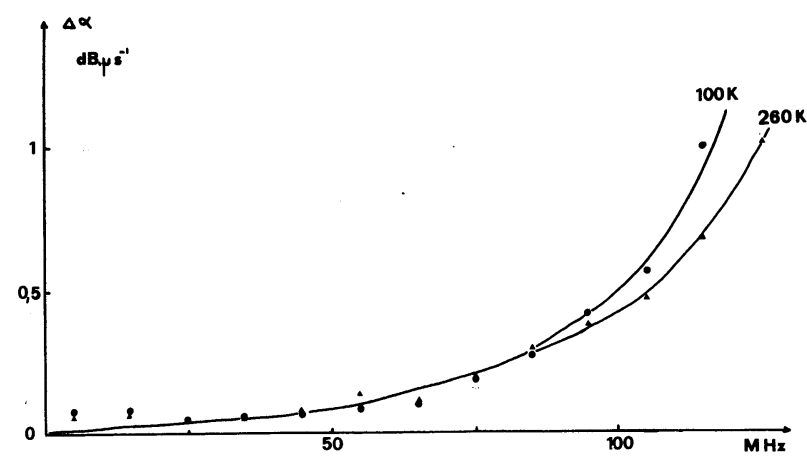

Fig. 4. - Influence de la température sur la variation d'atténuation avec la fréquence.

[Influence of temperature on the attenuation variation with frequency.]

3.3 Effet De La CONTRAinte Quasi Statique. Pendant un essai de traction limité à la déformation élastique de l'échantillon nous avons mesuré la variation d'atténuation à différentes températures. 


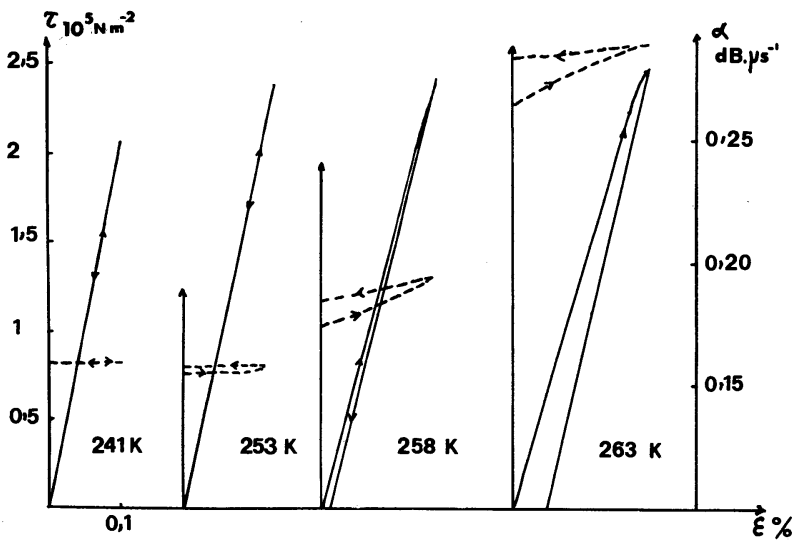

Fig. 5. - Variation de l'atténuation pendant l'application d'une contrainte quasi statique (Monocristal prédéformé de $3,4 \%$ à $265 \mathrm{~K}$ ).

[Attenuation variation during the application of a quasi static stress (prestained single crystal $\varepsilon=3.4 \%$ at $265 \mathrm{~K}$ ).]

Pour un monocristal préalablement déformé plastiquement de $3,4 \%$ à $265 \mathrm{~K}$, les résultats sont rassemblés sur la figure 5. A basses températures il n'apparaît pas d'augmentation d'atténuation lors de la mise sous contrainte. Par contre dès $258 \mathrm{~K}$ une augmentation d'atténuation apparaît dès l'application de la contrainte mais cette augmentation est irréversible : en effet, après décharge de la contrainte quasi statique l'excès d'atténuation subsiste. Il faut toutefois noter que l'application de la contrainte a, dans ce cas, entraîné une légère déformation plastique.

4. Interprétation. - Aux fréquences ultrasonores, les défauts ponctuels ne sont généralement pas susceptibles de créer une atténuation mesurable. Par contre, les dislocations mises en mouvement par l'onde de contrainte ultrasonore induisent par leur déplacement, une dissipation d'énergie, donc une atténuation.

Nous avions déjà tenté de décrire ce phénomène de dissipation d'énergie par les dislocations en utilisant des modèles classiques de corde vibrante ou de décrochements géométriques [1].

En fait, le modèle de corde vibrante ne tient pas compte de l'aspect spécifique du mouvement des dislocations dans la glace (orientation des liaisons hydrogènes, forces de Peierls vraisemblablement importantes à basses températures...).

Quant au modèle de décrochements géométriques [3] il est nécessaire de limiter le déplacement des décrochements à la distance entre deux molécules d'eau non favorablement orientées [4].

Nous avons donc développé deux modèles qui nous semblent plus appropriés au cas de la glace.

4.1 MODÈlES DE DISSIPATION D’ÉNERGIE ULTRASONORE PAR LES DISLOCATIONS DANS LA GLACE. Il est maintenant bien connu que le désordre protonique constitue un obstacle au mouvement des dislocations dans la glace $[5,6]$. Dans le cas des grandes vitesses (situation correspondant aux expériences faites avec les ultrasons), un déplacement des défauts linéaires sur une distance dépassant $b$ ou quelques $b$ impliquerait la formation de défauts rotationnels puisque les molécules d'eau n'auraient pas le temps de se réorienter (cf. les modèles reposant sur cette idée $[4,7,8])$. On doit donc considérer qu'une dislocation dans un cristal de glace se déplace à courte distance dans un puits de potentiel ; la hauteur des parois de ce puits correspond alors tout simplement à l'enthalpie de formation des défauts rotationnels qui se formeraient lors d'un déplacement à plus longue distance de cette dislocation. Dans ces conditions, la notion de tension de ligne (modèle de corde vibrante [9]) comme celle d'interaction entre décrochements (modèle de décrochements [3]) ne peuvent être retenues. Compte tenu des travaux récents sur le comportement dynamique des dislocations dans la glace, il est logique de considérer, à basses températures, un mouvement de décrochements, tandis qu'à hautes températures $(T \geqslant 240 \mathrm{~K})$ l'extension du cœur doit être pris en compte [10].

4.1.1 Modèle de décrochement modifié. - Nous n'envisagerons donc plus l'interaction entre décrochements comme dans le modèle de Suzuki et Elbaum [3] mais nous considérons que chaque décrochement se déplace dans un puits de potentiel lié à la nécessité de créer un défaut rotationnel pour un déplacement égal en moyenne à $2 b$.

L'équation du mouvement d'un décrochement s'écrira dans ces conditions

$$
m \frac{\partial^{2} U_{\mathrm{i}}}{\partial t^{2}}+B_{\mathrm{d}} \frac{\partial U_{\mathrm{i}}}{\partial t}+D_{\mathrm{d}} U_{\mathrm{i}}=\sigma b a
$$

$D_{\mathrm{d}} U_{\mathrm{i}}$ étant la force résultant du déplacement du décrochement dans le puits de potentiel, $m$ la masse d'un décrochement, $B_{\mathrm{d}}$ le terme de frottement visqueux, $a$ la distance entre collines de Peierls.

Lorsqu'un décrochement se déplace de $2 b$ (valeur moyenne) l'incrément d'énergie $\Delta E_{\max }$ correspond à l'enthalpie de création d'un défaut rotationnel

$$
\Delta E_{\max }=E_{\mathrm{F}} .
$$

Dans l'approximation d'un puits de potentiel parabolique l'incrément d'énergie $\Delta E$ correspondant au déplacement $U_{\mathrm{i}}$ d'un décrochement est donné par :

$$
\Delta E=E_{\mathrm{F}}\left(\frac{U_{\mathrm{i}}}{2 b}\right)^{2}
$$

et la force de rappel est :

$$
|\Delta F|=\frac{\mathrm{d} \Delta E}{\mathrm{~d} U_{\mathrm{i}}}=\frac{E_{\mathrm{F}} U_{\mathrm{i}}}{2 b^{2}}
$$

d'où

$$
D_{\mathrm{d}}=\frac{E_{\mathrm{F}}}{2 b^{2}} \text {. }
$$


En prenant une solution $U_{\mathrm{i}}=U_{\mathrm{i} 0}^{*} \exp i \omega t$, on a :

$$
U_{\mathrm{i} 0}^{*}=\frac{\sigma b a}{\left(\frac{E_{\mathrm{F}}}{2 b^{2}}-m \omega^{2}\right)+i \omega B_{\mathrm{d}}} .
$$

Introduisons la complaisance complexe :

$$
J^{*}=\frac{\varepsilon^{*}}{\sigma} \quad\left(\text { avec } \varepsilon^{*}=\text { déformation complexe }\right) .
$$

Rappelons que :

$$
\varepsilon^{*}=\varepsilon_{\text {dislocation }}^{*}+\varepsilon_{\text {ellastique }}
$$

avec $\varepsilon_{\text {dislocation }}^{*}=N U_{\mathrm{i}}^{*} a b(N=$ nombre de décrochements).

La résolution de l'équation différentielle initiale permet d'exprimer l'atténuation par le résultat :

$$
\alpha_{{\mathrm{dB} \mu \mathrm{s}^{-1}}^{-1}}=8.68 \times 10^{-6} \frac{G b^{2} a^{2}}{2 \Pi m} \frac{N \omega^{2} \beta_{1}}{\left(\omega_{0}^{2}-\omega^{2}\right)^{2}+\omega^{2} \beta_{1}^{2}}
$$

en posant $\quad \omega_{0}^{2}=\frac{E_{\mathrm{F}}}{2 b^{2} m} \quad$ et $\quad \beta_{1}=\frac{B_{\mathrm{d}}}{m}$

avec $G$ : module de cisaillement de la glace.

$\omega_{0}$ atteint une valeur maximale de $10^{12} \mathrm{~s}^{-1}$. L'anomalie de vitesse associée est :

$$
\frac{\Delta V}{V}=-\frac{G b^{2} a^{2}}{m} \frac{N\left(\omega_{0}^{2}-\omega^{2}\right)}{\left(\omega_{0}^{2}-\omega^{2}\right)^{2}+\omega^{2} \beta_{1}^{2}} .
$$

4.1.2 Modèle de cour étendu. - A haute température $(T \geqslant 240 \mathrm{~K})$ le cœur des dislocations dans la glace pourrait être étendu : un modèle de cœur non cristallin décrit par Perez et al. [10] rend compte de cette extension. La notion de décrochements doit alors être abandonnée.

On considère alors que les dislocations vibrent sous l'action de la contrainte ultrasonore dans un puits de potentiel sans qu'intervienne la tension de ligne. L'origine physique de ce puits de potentiel est, d'une part, l'orientation des liaisons, d'autre part, la variation d'énergie d'extension du cœur des dislocations.

En utilisant un formalisme similaire de celui de la corde vibrante, l'équation d'équilibre s'écrit :

$$
\frac{A \partial^{2} x}{\partial t^{2}}+\frac{B \partial x}{\mathrm{~d} t}+D x=\sigma b
$$

$D x=$ force de rappel résultant du déplacement de la dislocation dans le puits de potentiel.

Deux cas limites sont à envisager quant à la nature de la force de rappel.

a) Orientation des liaisons. — L incrément d'énergie, lorsque l'unité de longueur de dislocation se déplace de $b$, correspond à l'énergie de création de défauts rotationnels dont le nombre moyen par unité de longueur est $1 / 2 b$.

$$
\Delta E_{\max }=\frac{E_{\mathrm{F}}}{2 b} .
$$

Pour un puits parabolique on a, comme précédemment, un incrément d'énergie $\Delta E$ correspondant à un déplacement $x$ de la dislocation ;

et

$$
\begin{gathered}
\Delta E=\frac{E_{\mathrm{F}} x^{2}}{2 b^{3}} \\
D=\frac{E_{\mathrm{F}}}{b^{3}} .
\end{gathered}
$$

b) Variation de l'énergie d'extension du cour. Dans ce cas l'incrément d'énergie est la variation d'énergie libre du cœur étendu de la dislocation. D'après les expressions donnant $r^{*}$, rayon du cœur étendu, dans le modèle décrit par Perez et al. [10] on a :

$$
\begin{aligned}
\Delta E_{\max } & =\Delta G \\
\Delta E & =\frac{\Delta G x^{2}}{\left(r^{*}-r_{0}\right)^{2}}
\end{aligned}
$$

$\left(r_{0}=\right.$ rayon de cœur non étendu) et

$$
D=\frac{2 \Delta G}{\left(r^{*}-r_{0}\right)^{2}}
$$

Dans les deux cas, il faut noter que l'amplitude du mouvement ne peut dépasser une valeur de $b$ pour lequel le problème de la rupture et de la reconstitution des liaisons se pose. Un calcul analogue au $1^{\mathrm{er}}$ modèle donne :

$$
\alpha_{{\mathrm{dB} \mu \mathrm{s}^{-1}}}=8,68 \times 10^{-6} \rho_{\mathrm{d}} \frac{G b^{2}}{2 \Pi A} \frac{\beta_{2} \omega^{2}}{\left(\omega_{0}^{2}-\omega^{2}\right)^{2}+\omega^{2} \beta_{2}^{2}}
$$

avec

$$
\begin{gathered}
\omega_{0}^{2}=\frac{D}{A} \text { et } \beta_{2}=\frac{B}{A} \\
\text { et } \quad \frac{\Delta V}{V}=\rho_{\mathrm{d}} \frac{G b^{2}}{A} \frac{\omega_{0}^{2}-\omega^{2}}{\left(\omega_{0}^{2}-\omega^{2}\right)^{2}+\omega^{2} \beta_{2}^{2}} .
\end{gathered}
$$

Dans les deux cas limites, le calcul de $\omega_{0}$ donne :

- orientation des liaisons $\omega_{0} \simeq 10^{11} \mathrm{~s}^{-1}\left(r^{*} \simeq 4 \mathrm{~b}\right.$ à $260 \mathrm{~K}[10])$

- variation d'énergie du cœur $\omega_{0} \simeq 10^{10} \mathrm{~s}^{-1}\left(r_{0} \mathrm{de}\right.$ l'ordre de $b$ ).

Nous considérerons dans la suite des valeurs de $\omega_{0}$ comprises entre ces 2 valeurs.

4.2 Discussion : ApPlicabilité DES MODÈles AUX RÉSULTATS EXPÉRIMENTAUX. - Une difficulté d'application des modèles développés sera évidemment de définir correctement la forme du puits de potentiel dans lequel bougent les décrochements ou la dislo- 


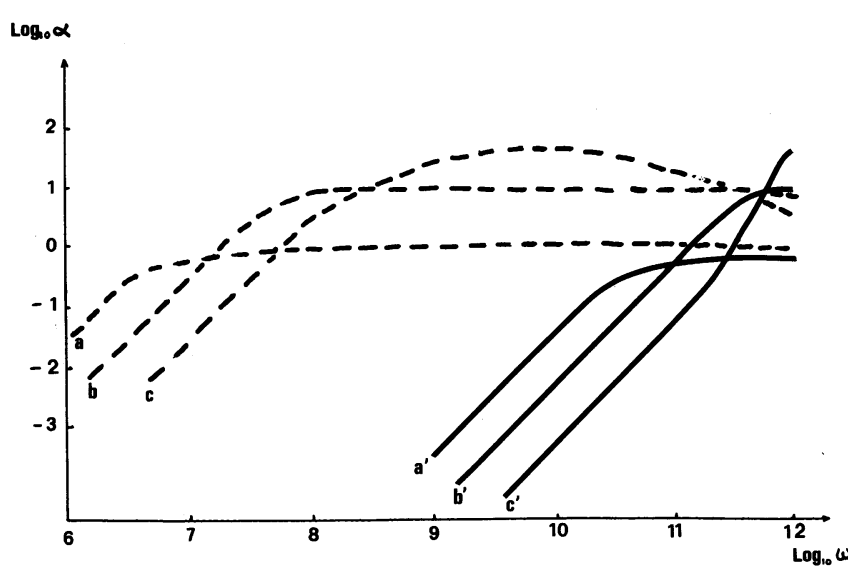

Fig. 6. - Variations théoriques de l'atténuation avec la fréquence :
a) $\omega_{0}=10^{10} \mathrm{~s}^{-1} ; \quad B=10^{-5} \mathrm{~S} . \mathrm{I}$
b) $\omega_{0}=10^{10} \mathrm{~s}^{-1} ; \quad B=10^{-6}$ S.I.
c) $\omega_{0}=10^{10} \mathrm{~s}^{-1} ; \quad B=10^{-7} \mathrm{~S}$.I.
a) $\omega_{0}=10^{12} \mathrm{~s}^{-1} ; \quad B_{\mathrm{d}}=5 \times 10^{-15}$ S.I
$\left.b^{\prime}\right) \omega_{0}=10^{12} \mathrm{~s}^{-1} ; \quad B_{\mathrm{d}}=5 \times 10^{-16}$ S.I
$\left.c^{\prime}\right) \omega_{0}=10^{12} \mathrm{~s}^{-1} ; \quad B_{\mathrm{d}}=5 \times 10^{-17}$ S.I.

[Theoretical variations of attenuation versus frequency :
a) $\omega_{0}=10^{10} \mathrm{~s}^{-1} ; \quad B=10^{-5}$ S.I
b) $\omega_{0}=10^{10} \mathrm{~s}^{-1} ; \quad B=10^{-6}$ S.I.
c) $\omega_{0}=10^{10} \mathrm{~s}^{-1} ; \quad B=10^{-7} \mathrm{~S}$.I.
$\left.a^{\prime}\right) \omega_{0}=10^{12} \mathrm{~s}^{-1} ; \quad B_{\mathrm{d}}=5 \times 10^{-15}$ S.I.
$\left.b^{\prime}\right) \omega_{0}=10^{12} \mathrm{~s}^{-1} ; \quad B_{\mathrm{d}}=5 \times 10^{-16}$ S.I.
c) $\omega_{0}=10^{12} \mathrm{~s}^{-1} ; \quad B_{\mathrm{d}}=5 \times 10^{-17}$ S.I.]

cation à cœur étendu. Des estimations de $\omega_{0}$ pour les deux modèles donnent des valeurs comprises entre $10^{10} \mathrm{~s}^{-1}$ et $10^{12} \mathrm{~s}^{-1}$ dans le cas de potentiels paraboliques. Cependant compte tenu de la grande directivité des liaisons dans la glace on peut envisager des puits de potentiel de forme plus complexe [11] modifiant ainsi les valeurs de $\omega_{0}$. Néanmoins, nous avons représenté sur la figure 6 les variations théoriques de l'atténuation en fonction de la fréquence pour les valeurs théoriques de $\omega_{0}$ évaluées dans le paragraphe précédent. La densité de dislocations $\rho_{\mathrm{d}}$ est prise égale à $10^{9} \mathrm{~m}^{-2}$ et le nombre de décrochements de l'ordre de $4 \times 10^{17} \mathrm{~m}^{-3}$ correspondant à un décrochement tous les $5 \mathrm{~b}$. Nous avons également fait varier les termes $B$ et $B_{\mathrm{d}}$ de frottement visqueux. On remarque une montée de $\alpha$ proportionnelle au carré de la pulsation $\omega$ jusqu'à une certaine valeur de celle-ci à partir de laquelle la variation est plus complexe.

Les résultats expérimentaux donnant l'atténuation en fonction de la fréquence (Fig. 4) ne montrent aucune différence entre les hautes et les basses températures sauf en ce qui concerne les hautes fréquences $(v>90 \mathrm{MHz})$. Nous sommes conduits à admettre que $\omega_{0}$ présente sensiblement la même valeur dans tout le domaine de température exploré, que l'on envisage un modèle de décrochements (basse température) ou un modèle de cœur étendu (haute température). Pour comparer les modèles avec nos points expérimentaux, nous avons donc pris pour $\omega_{0}$ la valeur moyenne $10^{11} \mathrm{~s}^{-1}$. La figure 7 représente la comparaison entre les variations théoriques et expérimentales de l'atténuation avec la fréquence.

Nous pouvons encadrer nos valeurs expérimentales en prenant d'une part les valeurs $B_{\mathrm{d}}=2 \times 10^{-15}$ S.I. et $B=5 \times 10^{-6}$.S.I. et d'autre part, une densité de dislocations donnée par

$$
5 \times 10^{9}<\rho_{\mathrm{d}}<5 \times 10^{10} \mathrm{~m}^{-2} \quad \text { (haute température) }
$$
ou

$2 \times 10^{18}<N<2 \times 10^{19} \mathrm{~m}^{-3} \quad$ (basse température).

Dans le cas du modèle haute température, la montée d'atténuation en $\omega^{2}$ couvre un domaine de fréquence moins étendu : cela correspondrait à une variation plus lente de l'atténuation à haute fréquence. Nos résultats semblent effectivement indiquer un tel comportement.

Notons que la relation d'équivalence $B_{\mathrm{d}} \simeq B . b$ entre les coefficients de frottement visqueux $B$ et

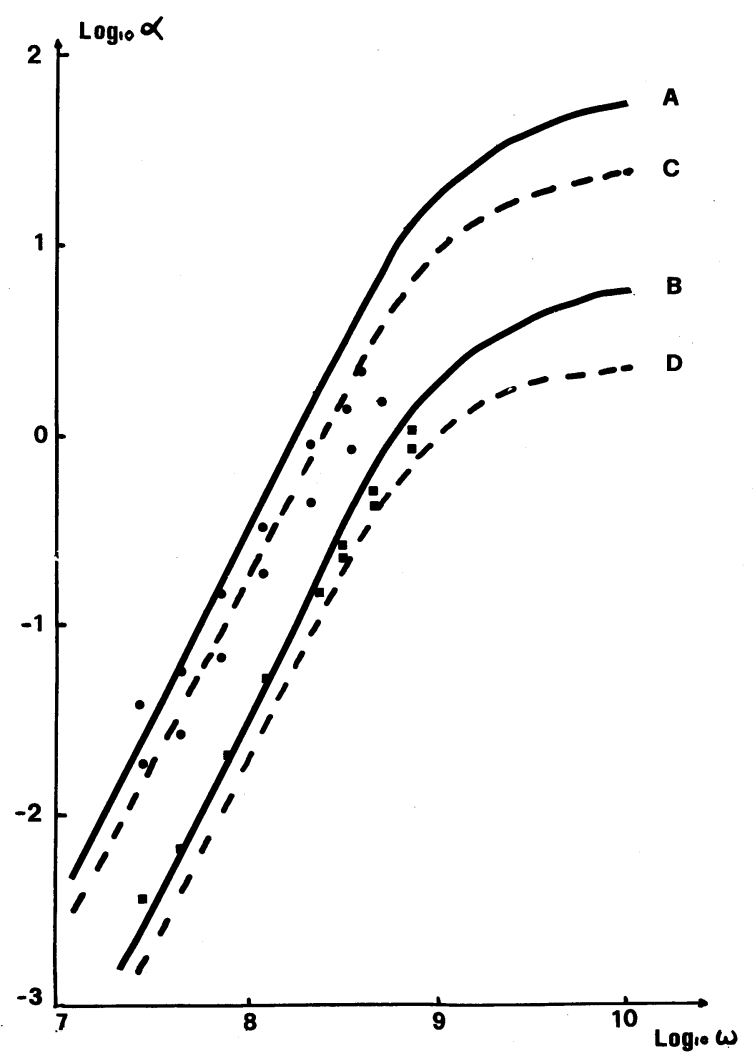

Fig. 7. - Comparaison des variations théoriques de l'atténuation avec la fréquence et des mesures expérimentales (ronds et carrés $\left.\omega_{0}=10^{11} \mathrm{~s}^{-1}\right)$
A) $N=2 \times 10^{19} \mathrm{~m}^{-3} ; \quad B_{\mathrm{d}}=2 \times 10^{-15}$ S.I
B) $N=2 \times 10^{18} \mathrm{~m}^{-3} ; \quad B_{\mathrm{d}}=2 \times 10^{-15}$ S.I.
C) $\rho_{\mathrm{d}}=5 \times 10^{10} \mathrm{~m}^{-2} ; \quad B=5 \times 10^{-6}$ S.I.
D) $\rho_{\mathrm{d}}=5 \times 10^{9} \mathrm{~m}^{-2} ; \quad B=5 \times 10^{-6}$ S.I.

[Comparison between theoretical and experimental variations of attenuation with frequency (circles and squares $\omega_{0}=10^{11} \mathrm{~s}^{-1}$ )

A) $N=2 \times 10^{19} \mathrm{~m}^{-3} ; \quad B_{\mathrm{d}}=2 \times 10^{-15}$ S.I.

B) $N=2 \times 10^{18} \mathrm{~m}^{-3} ; \quad B_{\mathrm{d}}=2 \times 10^{-15}$ S.I.

C) $\rho_{\mathrm{d}}=5 \times 10^{10} \mathrm{~m}^{-2} ; \quad B=5 \times 10^{-6}$ S.I.

D) $\rho_{\mathrm{d}}=5 \times 10^{9} \mathrm{~m}^{-2} ; \quad B=5 \times 10^{-6}$ S.I. $]$ 
$B_{\mathrm{d}}$ correspondant respectivement aux modèles de corde ou de décrochements est bien respectée. Il est intéressant de comparer la valeur de ces coefficients aux données de la littérature. L'hypothèse de l'interaction dislocation-phonon conduit à une valeur théorique de $10^{-6}$ S.I. $[12,13]$. En fait, on signale le plus souvent des valeurs expérimentales supérieures dans le cas de métaux [12]; au contraire, Suzuki et Elbaum envisagent pour le silicium $B_{\mathrm{d}}$ de l'ordre de $10^{-17} \mathrm{~S}$.I. valeur bien inférieure à celle évoquée dans le présent travail. Quant à la glace, il faut constater que la littérature ne donne, à notre connaissance, pas d'indications concernant indiscutablement $B$ : Van Devender et Itagaki [14] mentionnent bien la valeur $B=1,7 \times 10^{-7}$ mais le résultat est tiré de l'interprétation du pic de relaxation, habituellement observé avec la glace en terme de relaxation des dislocations alors que ce pic résulte très probablement de la réorientation des molécules d'eau; pour sa part Joncich, utilise $B$ de l'ordre de $10^{2}$ S.I., cette valeur de huit ordres de grandeur plus élevée correspond aux déplacements à longue distance des dislocations pour lesquels intervient le désordre protonique; les mécanismes s'opposant au mouvement des dislocations sont alors tout à fait différents comme l'indiquent les divers modèles proposés ces dernières années $[6,7,8,10]$. On peut donc conclure que nos résultats sont bien compatibles avec l'idée que les ondes ultrasonores peuvent, dans la glace, être amorties par un processus d'interaction dislocationsphonons.

Enfin, la légère augmentation de l'atténuation avec la température observée à $5 \mathrm{MHz}$ au-dessus de $250 \mathrm{~K}$ est vraisemblablement associée pour une part à l'amorce du phénomène de relaxation lié à la relaxation des molécules d'eau, et pour, d'autre part, à la modification de la structure de cœur des dislocations lorsque l'on approche la température de fusion [10].

Les résultats obtenus par application d'une contrainte quasi statique (Fig. 5) confirment également qu'un modèle de décrochements classiques [4] ne peut s'appliquer au-dessous de $258 \mathrm{~K}$. En effet, si tel était le cas on devrait nécessairement observer une diminution de l'atténuation lors de l'application de la contrainte quasi statique du fait de l'empilement des décrochements sur la ligne de dislocation comme l'ont montré Vincent et al. [15] dans le cas de l'aluminium.

Le résultat de la figure 5 est, au contraire, compatible avec le modèle proposé. En effet, quelle que soit la forme du puits de potentiel, le mouvement de la dislocation ou du décrochement restera limité à l'intérieur du puits. Ainsi, l'application d'une contrainte quasi statique conduit à un mouvement anharmonique des défauts linéaires : la position moyenne de ces défauts n'est plus le fond du puits mais il est facile de montrer que la diminution de l'amplitude du mouvement dans le sens correspondant à la contrainte quasi statique est sensible- ment compensée par l'augmentation de l'amplitude du mouvement opposé. La contrainte ultrasonore est atténuée par les dislocations de façon sensiblement identique que l'on applique ou non une contrainte quasi statique.

Il n'en est plus de même si l'on modifie la contrainte ultrasonore elle-même : un accroissement de l'amplitude de la contrainte ultrasonore donnera lieu à un accroissement de l'amplitude du mouvement de la dislocation. Mais les équations différentielles conduisant aux relations (1) et (2) étant linéaires du fait de notre hypothèse simplificatrice concernant la forme parabolique du puits, elles ne peuvent rendre compte d'un effet de la contrainte. En réalité, comme nous l'avons évoqué ci-dessus [11], ce puits de potentiel dans lequel se meuvent les défauts présente plus vraisemblablement un fond plus plat et des parois plus abruptes que ne l'indique la forme parabolique. Dans ces conditions, l'amplitude du mouvement des défauts variera moins vite que la contrainte : il en est donc de même de l'énergie dissipée devant l'énergie élastique de l'onde ultrasonore, entraînant par là une diminution de l'atténuation. Ainsi pourrait s'expliquer le résultat de la figure 3 .

Il est à noter également qu'une estimation de l'anomalie de vitesse dans le cas des 2 modèles donne des valeurs de $\Delta V / V$ comprises entre $10^{-6}$ et $10^{-5}$, ce qui est inférieure à la limite de détection de notre appareillage. Nos résultats indiquent au contraire une augmentation de la vitesse. Cet effet anormal avait déjà été discuté dans le cadre de l'hypothèse d'un effet de modification de la microstructure par déformation plastique (en particulier la concentration en défauts ponctuels pouvant entraîner une

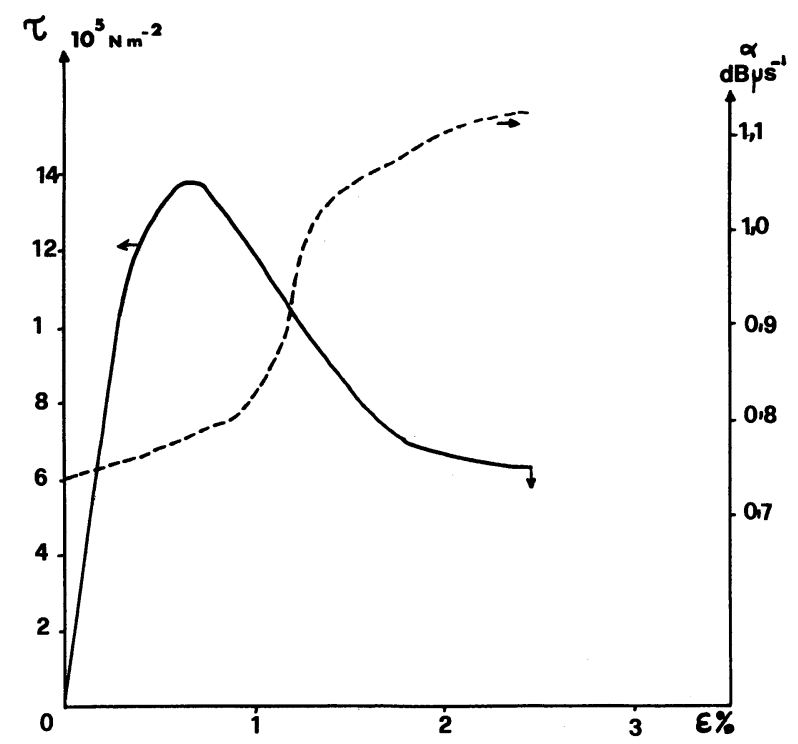

Fig. 8. - Variation de l'atténuation pendant la déformation plastique (compression) Monocristal Naturel. $T=259 \mathrm{~K}$. $V_{1}=5 \times 10^{-2} \mathrm{~mm} \cdot \min .^{-1}$. Fréquence de mesure $5 \mathrm{MHz}$.

[Attenuation variation during plastic deformation (compression). Natural single crystal. $T=259 \mathrm{~K} . V_{\mathrm{t}}=5 \times 10^{-2} \mathrm{~mm} \cdot \mathrm{min}^{-1}$. Frequency $5 \mathrm{MHz}$.] 
augmentation de la densité et de la rigidité du réseau [16]).

5. Application. - Nous avons appliqué le modèle " haute température " à des expériences de mesures d'atténuation pendant la déformation plastique même. L'idée principale de l'analyse de nos résultats a été de reconstituer de façon théorique les courbes effortdéformation en appliquant le modèle de Johnston [17] au cas de la glace. Nous nous sommes servis d'une part de l'expression de la vitesse des dislocations dans la glace dans le cas du cœur étendu [10], d'autre part de l'évaluation de la variation de densité de dislocations mobiles au cours de l'essai à l'aide de nos mesures d'atténuation effectuées pendant la déformation plastique.

La figure 8 montre, à titre d'exemple, la variation d'atténuation pendant un essai de compression. On doit noter que ce résultat, relatif à une glace naturelle pour laquelle on observe des défauts ou inclusions macroscopiques, correspond à un niveau d'atténuation élevée, dès l'origine mais la valeur de la variation d'atténuation reste comparable à celle observée dans le cas de glaces artificielles.

La vitesse de déformation est donnée par l'expression d'Orowan :

$$
\dot{\varepsilon}=\rho_{\mathrm{d}}(\varepsilon) \cdot b \cdot v_{\mathrm{d}}(\tau)
$$

où $\rho_{\mathrm{d}}$ est fonction de la déformation et la vitesse des dislocations $v_{\mathrm{d}}$ est fonction de la contrainte.

La vitesse des dislocations mobiles est donnée par l'expression [10] :

$$
v_{\mathrm{d}}(\tau)=C_{1} \tau^{1 / 2} \sinh C_{2} \tau^{1 / 2}
$$

avec

$$
\begin{aligned}
C_{1}=\frac{4}{9 \Pi} \frac{1}{\tau_{0}} \frac{b^{4}}{\left(r^{*} b\right)^{3 / 2} G^{1 / 2}} & \exp -\frac{2}{3}\left(\frac{E}{k T}\right)^{2}= \\
& =5.66 \times 10^{-9} \text { à } 260 \mathrm{~K}
\end{aligned}
$$

et

$$
C_{2}=\frac{\left(b r^{*}\right)^{3 / 2} G^{1 / 2}}{2 k T}=2,48 \times 10^{-3} \text { à } 260 \mathrm{~K}
$$

L'application du modèle de Johnston [17] conduit à l'équation différentielle :

$$
\frac{K}{A} f_{\mathrm{s}}-\frac{b K}{A} f_{\mathrm{s}} \frac{L_{0}}{V_{\mathrm{t}}} \rho_{\mathrm{d}}(\varepsilon) C_{1} \tau^{1 / 2} \sinh C_{2} \tau^{1 / 2}=\frac{\mathrm{d} \tau}{\mathrm{d} y}
$$

où $K$ est la raideur de la machine $\left(=2,8 \times 10^{6} \mathrm{~N} \mathrm{~m}^{-1}\right)$.

Nous considérons que la déformation élastique totale est due essentiellement à la déformation de la machine (à $5 \%$ près).

$A$ : est la section de l'éprouvette

$f_{\mathrm{s}}$ : le facteur de Schmidt

$L_{0}$ : la longueur initiale $\left(=40 \times 10^{-3} \mathrm{~m}\right)$

$V_{\mathrm{t}}$ : la vitesse de la traverse (de $5 \times 10^{-3} \mathrm{~mm} . \mathrm{min}^{-1}$ à $\left.5 \times 10^{-2} \mathrm{~mm} . \mathrm{min}^{-1}\right)$

$y$ : le déplacement de la traverse.
L'ajustement de la courbe théorique avec la courbe expérimentale effort-déformation permet de définir la valeur initiale $\rho_{\mathrm{d}}^{0}$ de la densité de dislocations mobiles et le facteur de proportionnalité $n$ entre la densité de dislocations créées lors de la déformation plastique et l'accroissement d'atténuation $\Delta \alpha$ qui en résulte :

$$
\rho_{\mathrm{d}}=\rho_{\mathrm{d}}^{0}+n \Delta \alpha
$$

Un tel ajustement est représenté sur la figure 9 à partir du résultat de la figure 8 .

Il est à remarquer que ces deux paramètres sont déterminés, d'une certaine manière, de façon indépendante : $\rho_{\mathrm{d}}^{0}$ dépend essentiellement des caractéristiques du maximum de la courbe effort-déformation, alors que $n$ correspond surtout à la partie suivante de cette même courbe.

Une telle reconstitution des courbes effort-déformation obtenues dans le cas des monocristaux de glace avait déjà été faite par Jones et Glen [18]; l'ajustement entre leur courbe expérimentale et celle calculée était meilleur que celui montré sur la figure 9 mais, d'une part, les auteurs disposaient d'un paramètre supplémentaire $\left(v_{\mathrm{d}}(\tau)\right.$ étant pris égal à $(\tau / D)^{p}$ avec $D$ et $p$ paramètres) et d'autre part le coefficient multiplicatif des dislocations avait été arbitrairement considéré comme étant identique à celui mesuré dans le cas du LiF.

D'un ensemble d'essais effectués sur des monocristaux de glace, naturelle ou artificielle, ne contenant pas trop de dislocations, sollicités soit en compression, soit en traction (l'analyse étant dans ce dernier cas moins satisfaisante du fait de l'hétérogénéité prononcée de la déformation plastique et donc de la distribution des dislocations), nous avons tiré les valeurs de $\rho_{\mathrm{d}}^{0}$ et de $n$ indiquées dans le tableau $I$. Ces valeurs sont comparées respectivement à l'estimation de $\rho_{\mathbf{d}}^{0}$ faite à partir d'observation en topo-

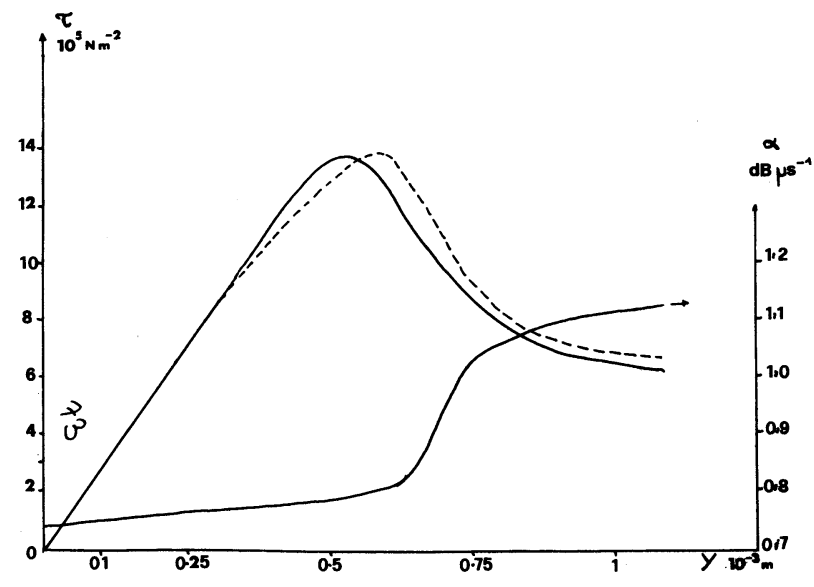

Fig. 9. - Reconstitution de la courbe effort-déformation (tirets). $\rho=10^{8}+8 \times 10^{9} \alpha$. Monocristal naturel $T=259 \mathrm{~K}$. Fréquence : $5 \mathrm{MHz}$.

[Building of stress-strain curve (doted curve). $\rho=10^{8}+8 \times 10^{9} \alpha$; natural single crystal. $T=259 \mathrm{~K}$. Frequency $5 \mathrm{MHz}$.] 
Tableau I.

$$
\begin{aligned}
& \text { A partir des courbes Observation Valeurs théoriques } \\
& \text { expérimentales topo } X \text { (relation (2)) } \\
& \rho_{\mathrm{d}}^{0} \quad 10^{8} \mathrm{à} 5 \times 10^{8} \mathrm{~m}^{-2} \\
& 0,8 \text { à } 18 \times 10^{10} \mathrm{~m}^{-2} \\
& \left(\mathrm{~dB} \mu \mathrm{s}^{-1}\right)^{-1}
\end{aligned}
$$

jusqu'à s'annuler quand $\varepsilon_{\mathrm{p}}$ augmente, ce qui est tout simplement caractéristique d'un régime dynamique.

6. Conclusion. - Nous avons décrit des modèles de dissipation d'énergie ultrasonore dans le cas de la glace. Ces modèles prennent en compte l'aspect spécifique des dislocations dans la glace; ils sont en relativement bon accord avec les mesures d'atténuation effectuées et conduisent à une évaluation du coefficient d'amortissement $B$ des dislocations par les phonons $\left(B \simeq 5 \times 10^{-6}\right.$ S.I. $)$.

L'utilisation de ces résultats et l'application du modèle de Johnston nous ont permis la reconstitution des courbes effort-déformation dans le cas de la glace. La cohérence de l'ensemble de nos résultats pourrait, contrairement à la conclusion de certains auteurs [14, 19], confirmer l'idée que les dislocations, dans la glace comme dans la plupart des solides cristallins, peuvent bien être à l'origine de l'amortissement des ondes ultrasonores par un processus d'interaction dislocations-phonons.

\section{Bibliographie}

[1] Tatibouet, J., Vassoille, R., Perez, J., J. Glaciol., Vol. 15, 73 (1975) 161-169.

[2] TATiBOuET, J., Thèse de Docteur Ingénieur, Université ClaudeBernard, Lyon (1978).

[3] Suzuki, T., Elbaum, C., J. Appl. Phys. 35 (1964) 1539-1544.

[4] Perez, J., Tatibouet, J., Vassollle, R., Gobin, P. F., Phil. Mag. 31, 5 (1975) 985-999.

[5] Glen, J. W., Phys. Kondens. Mater. 7 (1968) 43-51.

[6] Mai, C., Perez, J., Riviere, R., Tatibouet, J., Vassoille, R., Ann. Phys. 2 (1977) 91-118.

[7] Withworth, R. W., Paren, J. G., Glen, J. W., Phil. Mag. 33 (1976) 409-426.

[8] Frost, M. J., Goodman, D. J., Ashby, M. F., Phil. Mag. 33 (1976) 951-961.

[9] Granato, A. V., Lucke, K., J. Appl. Phys. 27 (1956) 583593.

[10] Perez, J., Mai, C., Vassohlle, R., J. Glaciol. Vol. 21, 85 (1978) 361-374.
[11] Eisenberg, D., Kauzmann, W., The structure and Properties of Water (Clarendon Press, Oxford), 1969, p. 247-248.

[12] Truell, R., Elbaum, C., Chick, B. B., Ultrasonic Methods in Solid State Physics (Academic Press, New York), 1969, p. 205.

[13] Hirth, J. P., Lothe, J., Theory of Dislocations (McGrawHill, New York) 1968, p. 193-196.

[14] Van Devender, J. P., Itagaki, K., Cold Regions Research and Engineering Laboratory Report, 243, 1973.

[15] Vincent, A., Perez, J., J. Phys.-Lett. 37 (1976) L 279-282

[16] Dantl, G., Physics of Ice, Riehl, Bullemer and Engelhardt Editors (Plenum Press, New York) 1969, p. 223.

[17] Johnston, W. G., J. Appl. Phys. 33 (1962) 2716-2730.

[18] JoNes, S. J., GLEN, J. W., J. Glaciol. 8 (1969) 463-473.

[19] JoNCICH, D. M., The Plastic behaviour of predeformed ice crystals, Thesis University of Illinois, 1976. 